\title{
Modeling of helix molecules formation on a surface of nanotube and inside it
}

\author{
Anna Belolipetskaya ${ }^{1}$, Nikita Lisitsa ${ }^{1}$, Igor Popov $^{1}$, Maria Faleeva $^{I^{*}}$ \\ ${ }^{1}$ ITMO University, Department of Higher Mathematics, 197101 St. Petersburg, Russia
}

\begin{abstract}
On-surface" synthesis of large molecules and assemblies is a great challenge in molecular electronics, biomedical devices, sensors, energy harnessing and catalysis. Usually, plane surfaces are used for these purposes. We study a possibility of macro molecules formation on the surface of nanotube and inside it. It can be used for synthesis of helix molecules, particularly, organic and biological. Nanotubes of different structures were considered. Point-like approximation is used for atoms. The Lennard-Jones 6-12 potential is taken as the interaction potential. Possibilities of helix molecules and ring-like molecules formations were shown. A hypothesis of such processes influence on the organic molecules formation during the early Earth history is suggested and discussed.
\end{abstract}

\section{Introduction}

In the fields of systems biology and systems chemistry, attaining compositional and topological diversity of macromolecules is fundamental for realizing complex functions [1]. "On-surface" synthesis with prefunctionalized molecules is a major research challenge in molecular electronics, biomedical devices, sensors, energy harnessing and catalysis [2-4]. Recently, it has led to specific one- and two-component products with remarkable efficiency [5-7].

There are four strategies now followed in the synthesis of large molecules and assemblies [8]: (i) controlled formation of covalent bonds, (ii) covalent polymerization, (iii) self-organization, (iv) molecular self-assembly. The third synthetic strategy abandons the covalent bond as a required connection between atoms and relies instead on weaker bonds, such as ionic bonds, hydrogen bonds, van der Waals interaction, to organize atoms, ions, or molecules into structures. Such method is used, e.g., for obtaining molecular crystals [9] or selfassembled monolayers [10]. We will discuss an approach close to the last two methods. Molecular self-assembly is the spontaneous association of molecules under equilibrium conditions into stable, structurally welldefined aggregates joined by noncovalent bonds A promising route to construction of functional systems with nanometre dimensions is the autonomous ordering and assembly of atoms and molecules on atomically well-defined surfaces [11]. Site-specific on-surface synthesis and on-surface formation of nanostructures were studied in many works (see, e.g., [12,13] and references therein).

In the present paper we investigate a possibility of helix structure (or long helix molecule) formation on the surface of nanotube and inside the nanotube. Nanotubes of different structures are considered. The most simple model for nanotube is based on zero-range potentials [14-16]. The mathematical background of the model is given by the theory of self-adjoint extensions of symmetric operators. We use more realistic model : a point-like approximation for atoms and the LennardJones 6-12 interaction potential:

$$
V(r)=4 \varepsilon\left(\left(\frac{\sigma}{r}\right)^{12}-\left(\frac{\sigma}{r}\right)^{6}\right)
$$

Here $\varepsilon$ is the depth of potential well, $\sigma$ is the distance for which the potential becomes zero, $r$ is the distance from the center.

\section{Model construction}

Different models are used for the description of nanotubes (see, e.g., [7] and references therein). We use the model of point-like potentials similar to that in [8] but with another interaction potential. We compute an energy surface which allows us to find local minima corresponding to preferable position of an atom disposed on the nanotube surface or inside it. The formula for the energy surface contains infinite series over all point-like potentials. This sum can be estimated by using of asymptotic approaches. Minima were found by numerical calculations.

The standard Lennard-Jones 6-12 potential with parameters $\sigma=2.28$ and $\varepsilon=51.2$ [1] without a cutoff distance was used to approximate interactions between a nanotube and a foreign particle. The potential isosurfaces were generated using the marching tetrahedra algorithm [2] on a tetrahedral grid, formed by tesellating each cell of a 3-dimensional cubic grid into six tetrahedra, with 
the cell's diagonal being a common edge. The potential's values on the grid vertices were precomputed and cach ed to speedup generation of multiple isosurfaces on the same data.

The most interesting pictures are observed when one considers the internal part of the nanotube. Figures show the position of energy minima as a dark area. The nanotube diameter and the structure of the nanotube (it is shown in the Figures) vary. A helix structure of the potential minimum set is observed in many cases. if one puts an atom in the nanotube, It will follow this helix way. Correspondingly, it is a place which is preferable for molecules formation. Thus, the nanotube plays a role of specific catalyst, ensuring the creation of helix-like molecular structure.

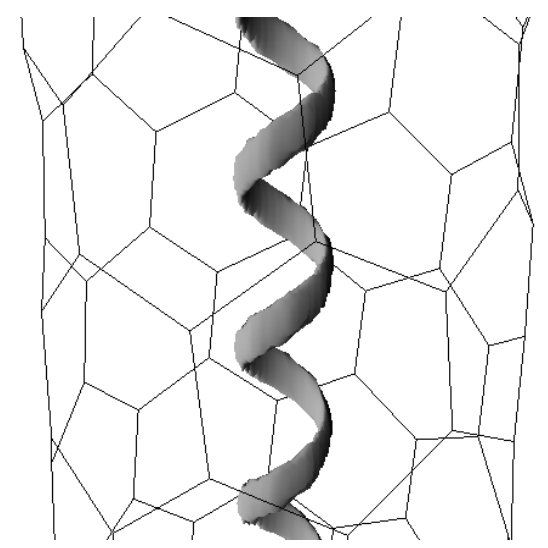

Fig. 1. Single helix structure of the potential minimum set..

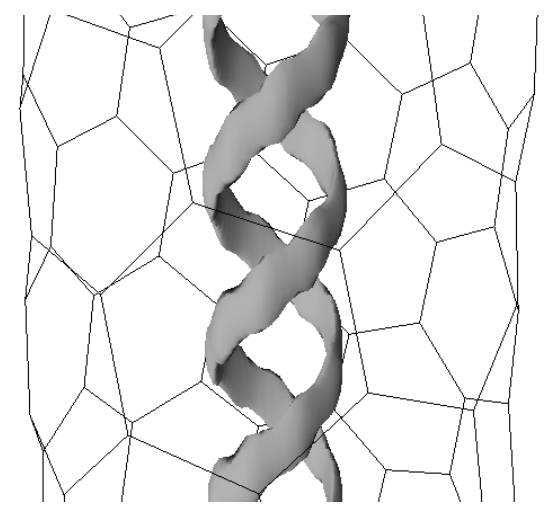

Fig. 2. Double helix structure of the potential minimum set.

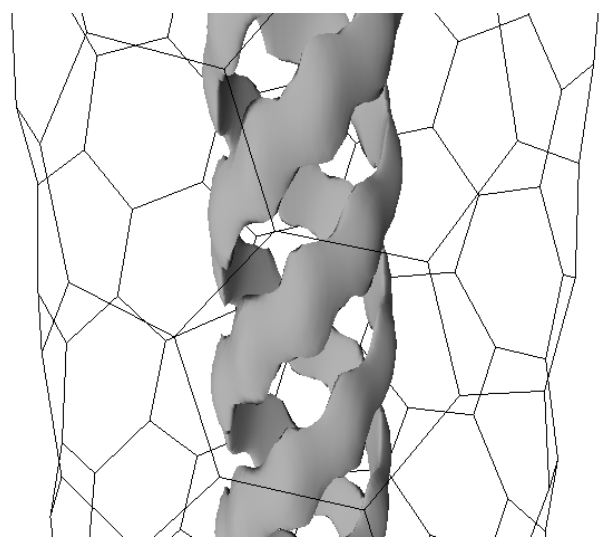

Fig. 3. Triple helix structure of the potential minimum set.

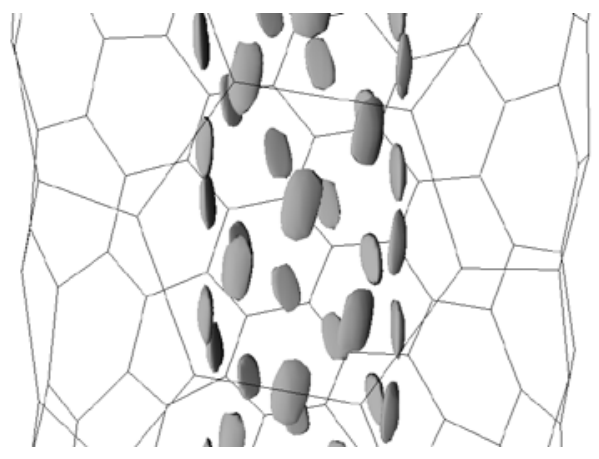

Fig. 4. Single helix structure of the potential minimum set.

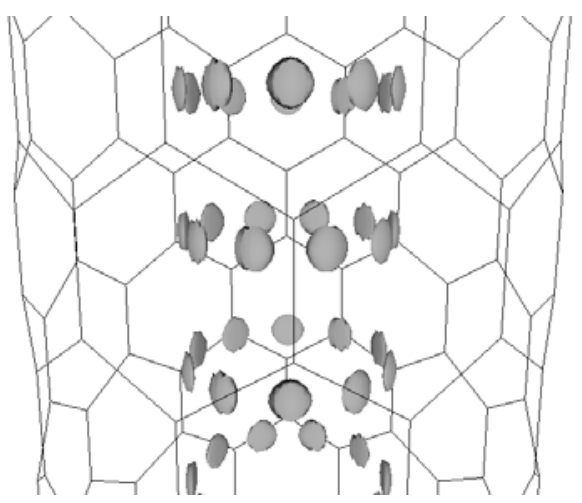

Fig. 5. Ring-like structure of the potential minimum set

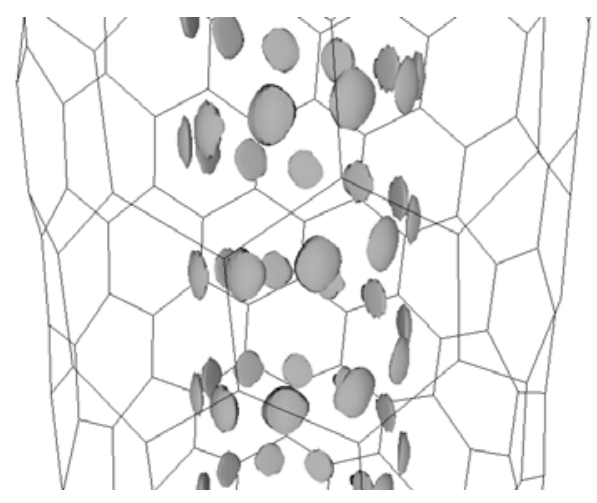

Fig. 6. Helix structure of the minima set.

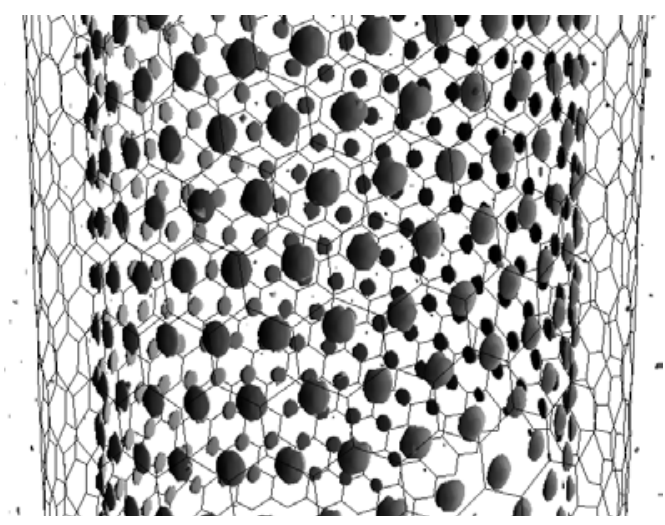

Fig. 7. . Helix structure of the minima set for large nanotube radius.

For the internal nanotube domain, wall confinement is an additional stabilizing factor stimulating macromolecules 
formation. As for the preferable positions for particles (atoms) at the external surface of the nanotube, one observes an analogous situation, but energy minima are not so deep as in the interior domain. The pictures are shown in the following figures (for various nanotube types).

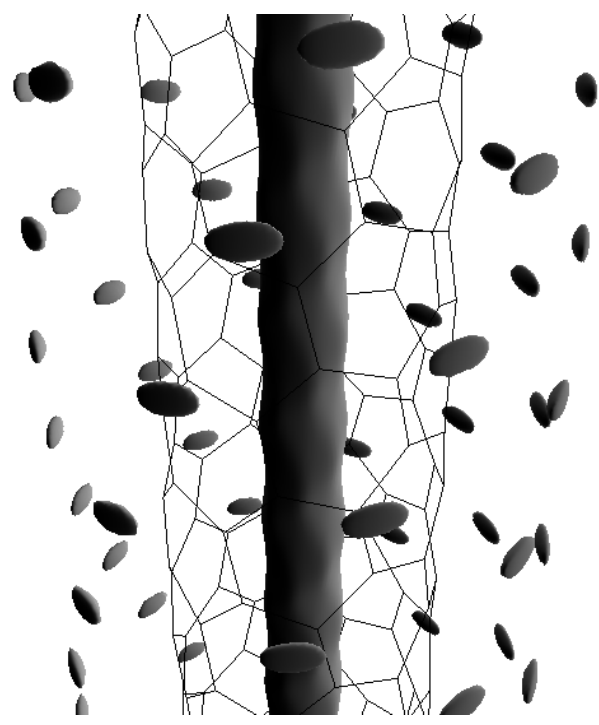

Fig. 8. . Helix structure of the external minima set for $(6,2)$ nanotube.

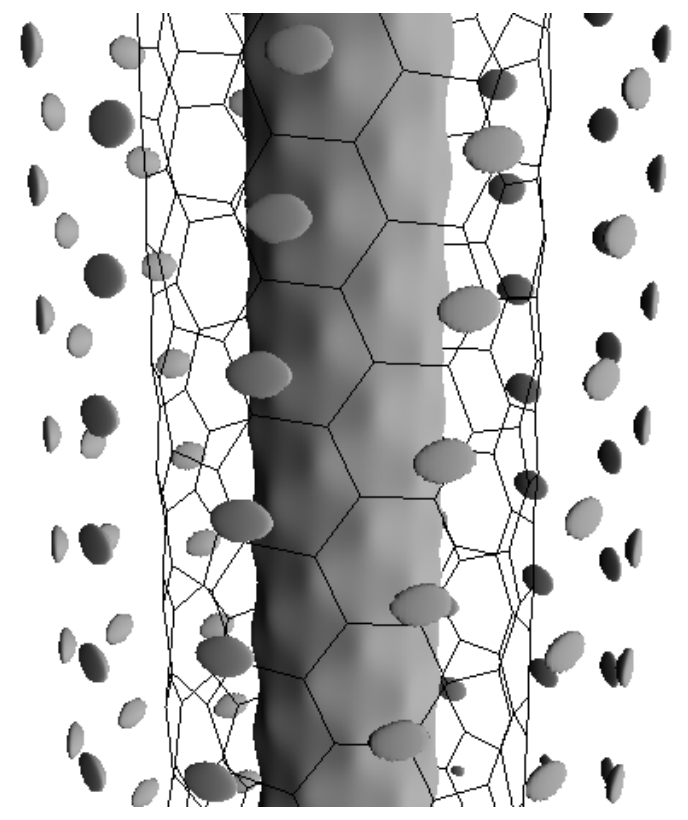

Fig. 9. . Helix structure of the external minima set for $(7,5)$ nanotube.

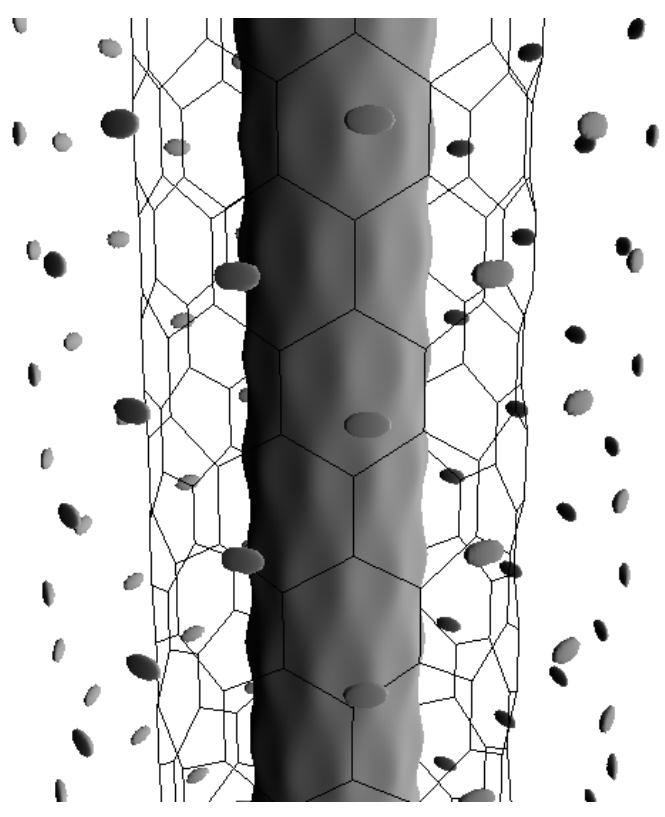

Fig. 10. . Helix (ring) structure of the external minima set for $(10,0)$ nanotube.

\section{Concluding remarks}

The obtained results show that one can use nanotubes for the process of helix structure macromolecules synthesis. In nanoelectronics, such molecules are useful for nano devices based on using of a magnetic field. Obviously, helix-like and ring-like molecules are interesting for pharmacy and bio engineering. We can also put a hypothesis concerning to the problem of life origin on the Earth, more precisely, to the process of organic molecules formation on the Earth. Namely, the process described above, can lead to formation (inside the nanotube or on its external surface) of a helix-like and ring-like molecules having biological nature. As for the nanotubes playing the role of a background, they appeared on the Earth essentially earlier than organic (biological) molecules. Correspondingly, such nanotubes can play a catalytic role for the organic molecules formation processes.

This work was partially financially supported by the Government of the Russian Federation (grant 074-U01), by the Ministry of Education and Science of the Russian Federation (GOSZADANIE 2014/190, Projects No 14.Z50.31.0031 and No. 1.754.2014/K)), by grants MK-5001.2015.1 and MK5161.2016.1 of the President of the Russian Federation, by grant 16-11-10330 of Russian Science Foundation.

\section{References}

1. R.F.. Ludlow, S. Otto. Chem. Soc. Rev 37, 101$108(2008)$

2. E. C. P.Smits, S. G. J. Mathijssen, , P. A. van Hal, S. Setayesh, T. C. T Geuns,.; K. A. H. A. Mutsaers, E. Cantatore,.; H. J Wondergem.; O.Werzer, R Resel, Nature, 455, 956-959 (2008) 
3. T.Aida, E.W. Meijer, S.I. Stupp. . Science, 335, 813-817 (2012)

4. M.Koepf, F. Cherioux, J.A.Wytko, J. Weiss. Coord. Chem. Rev. 256, 2872-2892 (2012)

5. L.Bartels. Nat. Chem. 2, 87-95 (2010)

6. J. Greenwood, H.A. Fruechtl, C.J. Baddeley. J. Phys. Chem. C, 117, 4515-4520 (2013)

7. Lackinger, M.; Heckl, W. M. J. Phys. D: Appl. Phys., 44, 464011 (2011).

8. G.M.Wintersides, J.P.Mathias, C.T.Seto. Science, 254, 1312-1319, (1991)

9. J.D.Wright. Molecular crystals. \{Cambriudge Univ. Press, Cambridge, 1987)

10. G.M.Wintersides, et.al. J. Am. Chem. Soc. 111, 321 (1989)

11. J. Barth, K Constantini, K.Kernth. Nature, 437, 67167 (2005)

12. C.J.Villagomez, T. Sasaki, J.M. Tour, L. Grill. J. Am. Chem. Soc. 111, 321 (1989)

13. S. Schlogl, V.M.Heckl, M.Lackinger. Surf. Sci. 606, 999-1004 (2012).

14. S. Albeverio and P. Kurasov. Singular perturbations of differential operators. Solvable Schrödinger type operators (London Mathematical Society, London, 2005

15. I.S. Lobanov, I.Yu. Popov, Nanosystems: Physics, Chemistry, Mathematics, 3(2), 6-28 (2012)

16. E.N.Grishanov, I.Y. Popov, Nanosystems: Physics, Chemistry, Mathematics, 7(5), 880-883 (2016) 